\title{
Disruption of Functional Brain Networks in Alzheimer's Disease: What Can We Learn from Graph Spectral Analysis of Resting-State Magnetoencephalography?
}

\author{
Willem de Haan, ${ }^{1,2}$ Wiesje M. van der Flier, ${ }^{2,3}$ Huijuan Wang, ${ }^{4}$ Piet F.A. Van Mieghem, ${ }^{4}$ \\ Philip Scheltens, ${ }^{2}$ and Cornelis J. Stam ${ }^{1}$
}

\begin{abstract}
In Alzheimer's disease (AD), structural and functional brain network organization is disturbed. However, many of the present network analysis measures require a priori assumptions and methodological choices that influence outcomes and interpretations. Graph spectral analysis (GSA) is a more direct algebraic method that describes network properties, which might lead to more reliable results. In this study, GSA was applied to magnetoencephalography (MEG) data to explore functional network integrity in AD. Sensor-level resting-state MEG was

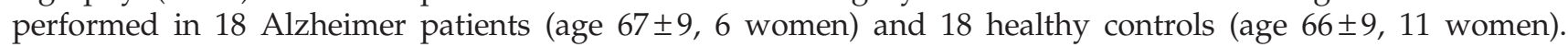
Weighted, undirected graphs were constructed based on functional connectivity analysis using the Synchronization likelihood, and GSA was performed with a focus on network connectivity, synchronizability, and node centrality. The main outcomes were a global loss of network connectivity and altered synchronizability in most frequency bands. Eigenvector centrality mapping confirmed the hub status of the parietal areas, and demonstrated a low centrality of the left temporal region in the theta band in AD patients that was strongly related to the mini mental state examination (global cognitive function test) score $(r=0.67, p=0.001)$. Summarizing, GSA is a theoretically solid approach that is able to detect the disruption of functional network topology in $\mathrm{AD}$. In addition to the previously reported overall connectivity losses and parietal area hub status, impaired network synchronizability and a clinically relevant left temporal centrality loss were found in AD patients. Our findings imply that GSA is valuable for the purpose of studying altered brain network topology and dynamics in AD.
\end{abstract}

Key words: dementia; eigenvector centrality; electrophysiology; functional connectivity; magnetoencephalography; network; neurophysiology; resting-state

\section{Introduction}

I N Alzheimer's disease (AD), the most prevalent form of dementia, imaging techniques have been successful in demonstrating local brain changes such as atrophy, hypometabolism, and protein deposition, but these phenomena do not express a straightforward relation with the gradually progressing severity of cognitive symptoms in AD (Pievani et al., 2011). Since cognition depends heavily on an efficient interaction between brain areas, changes in brain network connectivity might reflect cognitive decline more accurately. Both the investigation of the physical network wiring in the brain and the superimposed network dynamics ("functional" networks) may help in relating symptoms in AD to the underlying neurodegenerative processes.

In recent years, the graph theory has been increasingly used as a theoretical framework that describes brain network characteristics (Sporns, 2010). Graph theoretical studies in AD demonstrate the disruption of large-scale brain network integrity (de Haan et al., 2009; He et al., 2009; Lo et al., 2010; Sanz-Arigita et al., 2010; Stam et al., 2007, 2009; Supekar et al., 2008). However, applying graph theoretical concepts to neuroscience also poses methodological dilemmas. A growing number of measures is being developed (Rubinov and Sporns, 2010), and although the reproducibility of graph measures is good (Deuker et al., 2009), varying

\footnotetext{
${ }^{1}$ Department of Clinical Neurophysiology and Magnetoencephalography, VU University Medical Center, Amsterdam, The Netherlands.

${ }^{2}$ Department of Neurology, Alzheimer Center, VU University Medical Center, Amsterdam, The Netherlands.

${ }^{3}$ Department of Epidemiology and Biostatistics, VU University Medical Center, Amsterdam, The Netherlands.

${ }^{4}$ Faculty of Electrical Engineering, Mathematics and Computer Science, Delft University of Technology, Delft, The Netherlands.
} 
definitions can bias outcomes and interpretations. For example, many graph measures are directly dependent on network size and density, demanding arbitrary normalization or thresholding procedures (van Wijk et al., 2010).

Graph spectral analysis (GSA) is a technique that is known in fields such as mathematics, chemistry, and engineering for its powerful characterization of network features (Van Mieghem, 2011). In short, GSA investigates the spectrum of a network, which is the set of eigenvectors and corresponding eigenvalues that are mathematically derived from the adjacency or Laplacian matrix of the network. The spectrum not only contains considerable information on relevant network properties such as connectivity levels and resilience to damage, but also provides measures that are directly related to network dynamics, such as the spread of information throughout a network (Bonacich and Lloyd, 2001; Van Mieghem et al., 2009). Since the interaction between distant brain regions is essential for cognition, dynamical efficiency is probably an important aspect of large-scale brain network topology (Arenas et al., 2008). Two graph spectral measures described in this study, the spectral gap and eigenratio, make predictions about the dynamical behavior in a network based on its topology. Another relatively familiar graph spectral measure is the eigenvector centrality (EC), which is used to identify highly connected "hub" regions in networks (Bonacich, 2007; Lohmann et al., 2010). Since hub region vulnerability has been repeatedly reported in AD (Buckner et al., 2005; Stam et al., 2009), the further exploration of hub structure is very relevant, as it could point toward an explanation for this fundamental pathophysiologic phenomenon.

The deterministic nature of GSA and its solid theoretical background might make it a promising complement to the commonly used graph measures. We set out to investigate the AD-related changes in five spectral measures that describe network topology and hub status. To evaluate the clinical value of this approach, the relationship between regional EC and cognitive test scores was also examined. Our hypothesis was that, in addition to the previously reported loss-offunctional network connectivity, graph spectral measures would be able to detect impaired network synchronizability. In addition, we expected to find parietal hub region vulnerability and a corresponding decrease in the regional EC values in AD.

\section{Materials and Methods}

\section{Patients and controls}

The study involved 18 patients with a diagnosis of probable AD according to the NINCDS-ADRDA criteria (McKhann et al., 1984) who had been recruited from the Alzheimer Center of the VU University Medical Center. The AD patients were assessed according to a standard diagnostic workup, which involved history taking, physical and neurological examinations, an interview with a spouse or close family member, a neuropsychological assessment, blood tests, magnetic resonance imaging (MRI) of the brain, and electroencephalography. The diagnosis was made in a consensus meeting during which all the available clinical data were considered by a multidisciplinary team. The exclusion criteria for this study were active psychiatric or neurologic disease, or a mini mental state examination (MMSE) score below 16. Eighteen healthy controls, often spouses of patients,
Table 1. Subject Characteristics

\begin{tabular}{lccl}
\hline & Controls & Alzheimer patients & \\
\hline$N$ & 18 & 18 & \\
Age & $66 \pm 9$ & $67 \pm 9$ & $p=0.82$ \\
Gender (M/F) & $7 / 11$ & $12 / 6$ & $p=0.16$ \\
MMSE & $29 \pm 1$ & $23 \pm 1$ & $p<0.001$ \\
Education & $5 \pm 1$ & $5 \pm 1$ & $p=0.89$ \\
\hline
\end{tabular}

Education score is based on Verhage index (Verhage, 1965). Data are represented as mean \pm standard deviation unless indicated otherwise.

$N$, number of subjects; M, males; F, females; MMSE, mini mental state examination.

were also included. No structural (MRI) scans of the control subjects were made, but they were screened by a neurologist and underwent the same neuropsychological test battery as the patients. In both groups, the use of psychoactive medication was incidentally reported: antidepressants (specific serotonine reuptake inhibitors and tricyclic antidepressants, AD $n=3$ : controls $n=1$ ) and sleep medication (benzodiazepines, AD $n=1$ : controls $n=2$ ). Since AD patients had been diagnosed shortly before the magnetoencephalography (MEG) recording was performed, a few of them reported having used cholinesterase inhibitors (galantamine, $n=2$ ). Most frequent comorbidities were hypertension ( $\mathrm{AD} n=6$; controls $n=3$ ) and diabetes mellitus type $2(\operatorname{AD} n=4$ : controls $n=0)$. The main subject characteristics are summarized in Table 1.

Global cognitive functioning was assessed with the MMSE (Folstein et al., 1983). The level of education was classified according to the system of Verhage ranging from 1 to 7 (low to highly educated) (Verhage, 1965). The Local Research Ethics Committee approved the study, and all participants provided written informed consent. The subjects and recordings were identical to a recent graph theoretical study focusing on modularity (de Haan et al., 2012).

\section{MEG recording and post-processing}

Magnetic fields were recorded while the subjects were seated in a magnetically shielded room (Vacuumschmelze $\mathrm{GmbH}$, Hanau, Germany) using a 151-channel whole-head MEG system (CTF Systems, Inc., Port Coquitlam, BC, Canada). A third-order software gradient (Vrba and Robinson, 2001) was used after online band-pass filtering between 0.25 and $125 \mathrm{~Hz}$. The sample frequency was $625 \mathrm{~Hz}$. For technical reasons, two channels had to be omitted, leaving 149 channels for analysis. The subjects were measured during a no-task, eyes-closed condition. At the beginning and at the ending of the recording, the head position relative to the coordinate system of the helmet was recorded by leading small, alternating currents through three head-position coils attached to the left and right pre-auricular points and the nasion on the subject's head. During the recording, the head-position changes up to $\sim 1.5 \mathrm{~cm}$ were accepted. During the MEG recording, the subjects were instructed to close their eyes, stay awake, and reduce eye movements. In addition, they were instructed to just let their minds wander, and certainly not to perform specific cognitive tasks such as counting.

Typical artifacts were due to (eye) movements, swallowing, dental prosthetics, or drowsiness. For each subject, care was taken to select four artifact-free epochs of 4096 samples 
( $\sim 6.5 \mathrm{sec}$ ) by two of the investigators (W.D.H. and C.S.) who had been blinded to the diagnosis. All further analyses were performed in the following frequency bands: delta $(0.5-4 \mathrm{~Hz})$, theta $(4-8 \mathrm{~Hz})$, lower alpha $(8-10 \mathrm{~Hz})$, higher alpha (10$13 \mathrm{~Hz})$, beta $(13-30 \mathrm{~Hz})$, and gamma $(30-45 \mathrm{~Hz})$. All functional connectivity and graph analyses were separately performed for each epoch, and before the statistical analysis, the four epoch results of each person were averaged.

All functional connectivity and subsequent graph spectral analyses were performed with an in-house developed software (BrainWave version 0.8.68, CS. Software available at: http://home.kpn.nl/stam7883/brainwave.html). The graph spectral measures described next were implemented using an open access JAVA library called JAMA (www.cs.princeton .edu/introcs/95linear/Eigenvalues.java.html).

\section{Functional connectivity analysis}

Correlations between all pair-wise combinations of MEG channels were computed with the Synchronization Likelihood (SL). Mathematical details can be found in previous works (Montez et al., 2006; Stam and Dijk, 2002) and in the appendix; here, a brief description is provided. The SL is a general measure of the correlation or synchronization between two time series that is sensitive to linear as well as non- linear interdependencies. The basic principle of the SL is to divide each time series into a series of "patterns" (roughly, brief pieces of time series containing a few cycles of the dominant frequency) and to search for a recurrence of these patterns. The SL is then the probability that the pattern recurrence in time series $X$ coincides in time with the pattern recurrence in time series $Y$. The end result of computing the SL for all pair-wise combinations of channels is a square matrix (with 149 rows and columns, equal to the number of MEG channels), where each entry contains the resulting SL value of the sensor pair. This matrix is called the weighted (connections strengths or weights are included) adjacency or connectivity matrix $A$. Note that any connectivity measure could be used for this purpose. Since all connections in our network are bidirectional, the adjacency matrix is symmetrical along its diagonal axis.

\section{Graph spectral analysis}

In this section, a brief explanation of the concepts and measures used in this study is provided; for a more extensive technical background, please see (Bonacich and Lloyd, 2001; Brouwer and Haemers, 2011; Farkas et al., 2001; Newman, 2007; Van Mieghem, 2011). The multi-step procedure from the MEG recording to spectral analysis is summarized in Figure 1.
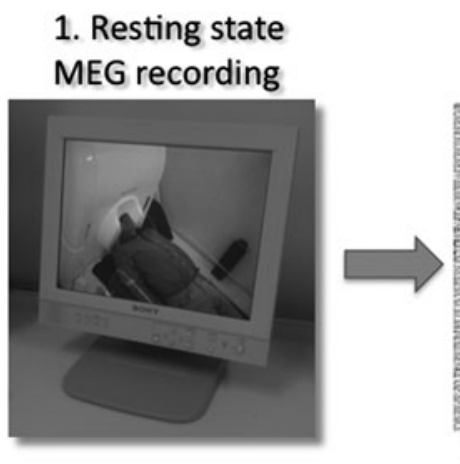

\section{Functional connectivity} analysis of MEG time series
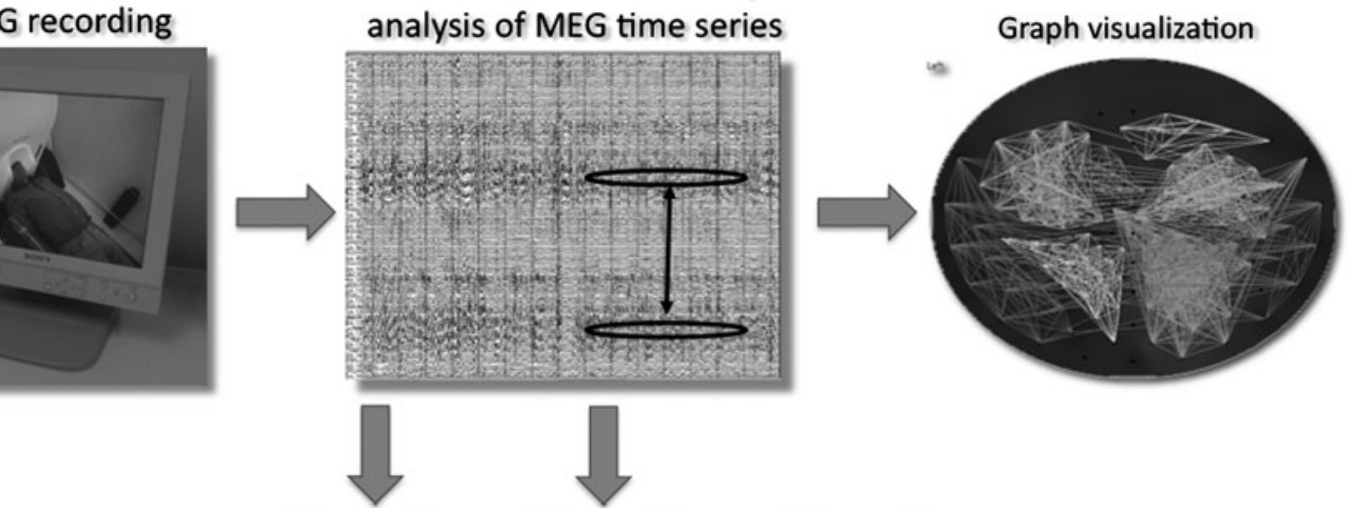

3. Matrix
construction
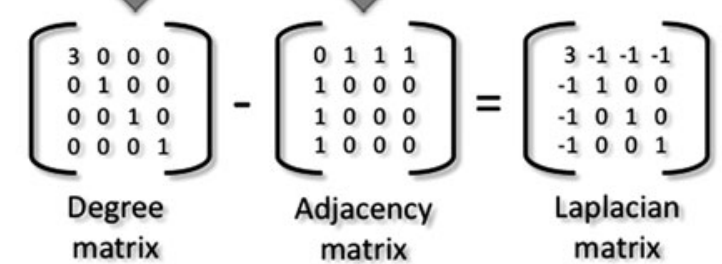

\section{Derivation of Laplacian matrix}

\section{Computation of graph spectra and spectral measures}

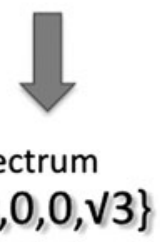

matrix

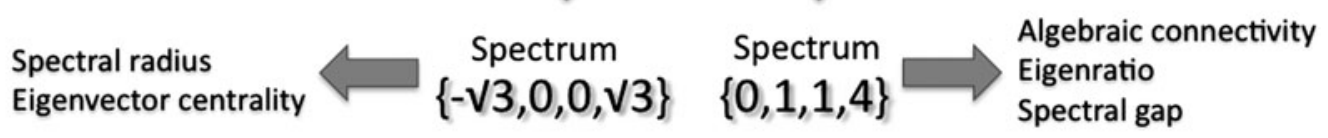

FIG. 1. Multi-step procedure from MEG recording to the computation of graph spectral measures. For this study, brain activity was recorded in an eyes-closed resting-state condition. Functional connectivity analysis was performed on 4 time segments (ca. $6.5 \mathrm{sec}$ ) per person. Subsequently, weighted functional brain networks were formed, and from the corresponding adjacency matrices, the Laplacian matrices were constructed. For each network, from both the adjacency and Laplacian matrices, the spectrum was calculated, and the eigenvalues from these spectra were used to compute various spectral measures. Spectral measures were then used in statistical analysis to compare group averages and correlations with cognition. It should be noted that once a network is constructed, its eigenvectors and eigenvalues will be determined. MEG, magnetoencephalography. 
Graph spectral measures are derived from the adjacency or Laplacian matrix $Q$. This is done by subtracting the adjacency matrix from the degree matrix $\Delta(Q=\Delta-A)$, which is the diagonal matrix with the nodal degrees (equal to the rowsum of the adjacency matrix); see Figure 1 for an example. The Laplacian matrix can be regarded as a manner of combining both connectivity and degree information (all relevant information) in the same matrix. Both the adjacency and the Laplacian matrix can be written in terms of their eigenvectors and corresponding eigenvalues, for example, $A=X \Lambda X^{T}$, where the matrix $X$ consists of all the eigenvectors in columns, and the diagonal matrix $\Lambda$ contains the corresponding eigenvalues. The spectral information ( $X$ and $\Lambda$ ), thus, contains the same information as the topology, or adjacency matrix (Van Mieghem, 2011). The spectrum of a graph can be regarded as a unique "fingerprint." Especially the different eigenvalues contain precise information on network properties, and can be used to quantitatively classify network topologies. Here, we briefly describe four graph spectral measures that contain meaningful information on the network as a whole (two derived from the adjacency matrix, two from the Laplacian matrix) and one measure with a more local focus.

\section{Global analysis}

The spectral radius $\lambda$ is the largest eigenvalue of the adjacency matrix, and it obeys $2 L / N \leq \lambda \leq d_{\max }$, where $N$ and $L$ are the number of links and nodes, respectively, and $d_{\max }$ is the maximum degree in the graph (Van Mieghem, 2011). For a fixed size $N$ of the network, the larger $\lambda$ is, the more links $L$, and the better connected the network (Brouwer and Haemers, 2011; Dvorák and Mohar, 2009). The inverse of the spectral radius equals the epidemic threshold in a network (Van Mieghem et al., 2009), and is proportional to the synchronization threshold of a network (Restrepo et al., 2005). It is also related to kappa, the ratio of the average squared degree and the average degree.

The spectral gap describes how fast a dynamic process in a network will converge to the steady state (Van Mieghem, 2011). It is equal to the difference between the two largest eigenvalues of the adjacency matrix. It should be noted that the spectral gap and the eigenratio (introduced next) are graph spectral measures that deal with synchronized states of a network, as opposed to the underlying synchronization measure between nodes (SL in this case) that is used to determine the connectivity matrix.

The algebraic connectivity, introduced by Fiedler in 1973, measures how difficult it is to tear a network apart. If the network is fully connected, then the algebraic connectivity is $>0$. The magnitude of the algebraic connectivity can also be regarded as a measure for network "robustness." The algebraic connectivity is equal to the second-smallest eigenvalue of the Laplacian matrix (Fiedler, 1973; Mohar, 1991; Van Mieghem, 2011).

The eigenratio expresses the stability of a synchronized state in a dynamical network. It is the ratio of the largest and the second-smallest eigenvalue of the Laplacian matrix. The smaller it is, the more stable the network synchronization (Arenas et al., 2008). In this study, we use its inverse (1/eigenratio) to obtain a value between 0 and 1 .

\section{Regional analysis}

The EC is a measure of the relative importance (or hub status) of a node within a network (Bonacich, 2007; Bonacich and
Lloyd, 2001). The most straightforward method of identifying hubs is by their degree centrality, which assigns a hub status to nodes with the highest number of connections (or the highest sum of all weighted connections). However, this measure only takes a node's direct connections into account. Popular alternative centrality measures that have a wider scope are the betweenness centrality and closeness centrality; however, they also have some drawbacks, such as their dependency on path length and considerable computational demands (Rubinov et al., 2009; Rubinov and Sporns, 2011). In contrast, the defining characteristic of the EC is that it takes into account both the degree of a node and the degrees of its neighbors. It, therefore, recognizes the fact that having important nodes as immediate neighbors makes a node more important in the network. Actually, the largest eigenvector component $i$ is a "dynamic" degree, where "dynamic" refers to all walks in the graph that traverse the node $i$. EC $x_{i}$ for node $i$ is the $i$ th component of the eigenvector corresponding to the largest eigenvalue of the adjacency matrix, and is equal to:

$$
x_{i}=\frac{1}{\lambda} \sum_{j=1}^{N} A_{i j} x_{j}
$$

where $\lambda$ is the largest eigenvalue of the adjacency matrix, $N$ is the total number of nodes, and $A$ is the adjacency matrix of the network. It should be noted that $x_{i}$ is proportional to the sum of weights of all the nodes connected to it. EC is calculated per node, but we averaged values over ten sensor groups (left and right frontal, temporal, central, parietal and occipital) to obtain a centrality distribution on a larger scale.

\section{Statistical analysis}

The statistical analysis was performed with SPSS for Mac (version 18.0). The normal distribution of all measures was checked with the Kolmogorov-Smirnov tests. For testing group differences with regard to spectral radius, spectral gap, algebraic connectivity, and eigenratio, we performed independent sample $t$-tests and nonparametric Mann-Whitney $U$ tests, which produced very similar results. We analyzed regional EC results using analysis of variance (ANOVA) for repeated measures (Greenhouse-Geisser corrected) with a group as a between-subjects factor, and hemisphere (left and right) and sensor region (frontal, central, temporal, parietal, and occipital) as within-subjects factors. Gender was included as covariate. Correlations between the regional EC values and MMSE scores were evaluated with the Pearson's test. Analyses were separately performed for all frequency bands. For all tests, a significance level of $\alpha \leq 0.05$ was used, and no correction for multiple comparisons was applied.

\section{Results}

\section{Global analysis}

The spectral radius was generally lower in AD patients, but this difference reached significance only in the gamma band $(p<0.01$, see Fig. 2). This indicates a higher network synchronizability threshold in the gamma band.

The spectral gap was lower in AD patients in all frequency bands except for the theta band, which was only significant in the gamma band ( $p<0.01$, see Fig. 3 ). This indicates that functional network dynamics in the gamma band will take longer to reach a steady, synchronized state in AD. 


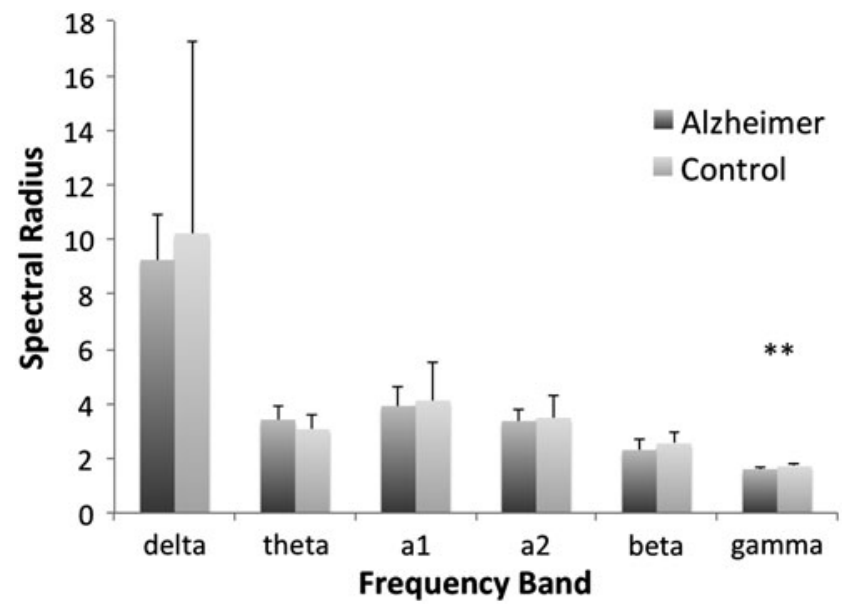

FIG. 2. Spectral radius results for the different frequency bands. Error bars indicate standard deviation. ${ }^{* *} p<0.01$ (uncorrected). AD, Alzheimer patient group; $\mathrm{C}$, control group; a1, lower alpha band; a2, higher alpha band.

The algebraic connectivity in $\mathrm{AD}$ patients was lower in the lower alpha $(p<0.05)$, beta $(p<0.01)$, and gamma bands $(p<0.01$, see Fig. 4). No differences were found in the remaining three bands. The decrease in multiple frequency bands can be interpreted as a loss of overall connectivity in AD.

The eigenratio was lower in the theta band $(p<0.05)$, and higher in the gamma band $(p<0.01)$ in $\mathrm{AD}$ when compared with controls (see Fig. 5). No differences were found in the other frequency bands. This implies that overall network synchronizability decreases in the theta band, but increases in the gamma band in AD.

\section{Regional analysis}

The ANOVA for repeated measures of regional EC results (see Table 2) showed no main effect of the group, but instead showed a main effect of the region in all bands except for the delta band. In the gamma band, a main effect of the hemisphere was found, but no effects were found in any of the other frequency bands. Moreover, higher alpha and gamma bands showed region-hemisphere interactions, indicating dis-

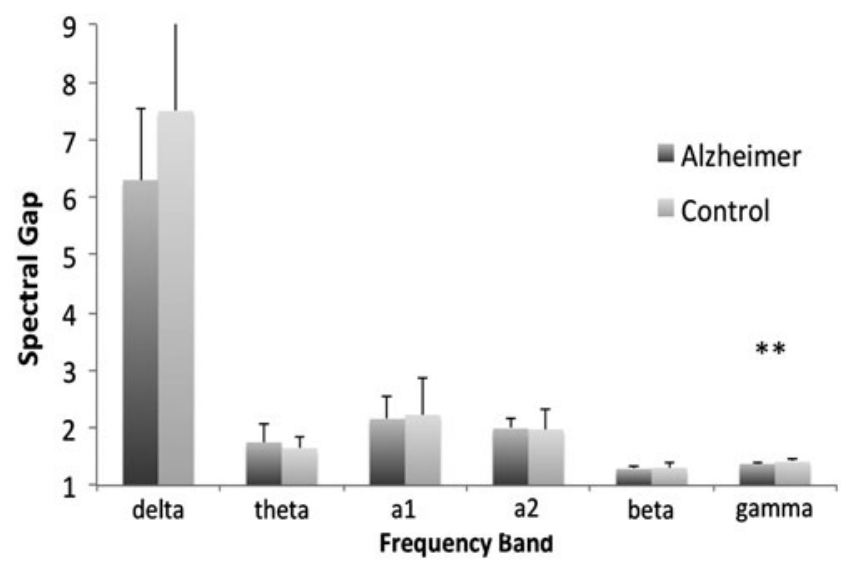

FIG. 3. Spectral gap results for the different frequency bands. Error bars indicate standard deviation. ${ }^{* *} p<0.01$ (uncorrected).

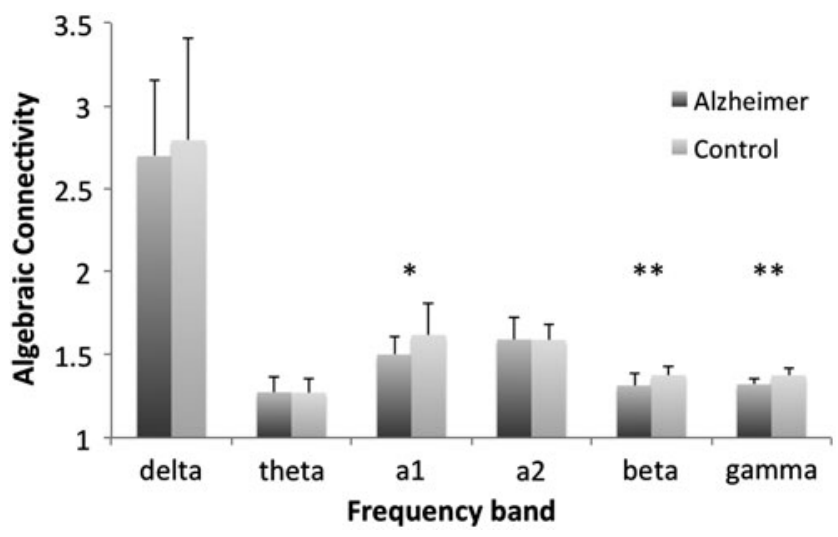

FIG. 4. Algebraic connectivity results for the different frequency bands. Error bars indicate standard deviation. ${ }^{*} p<0.05,{ }^{* *} p<0.01$ (uncorrected).

similar regional differences for each hemisphere in those bands. Region-group interactions were found in the theta and beta bands, as well as a hemisphere-group interaction in the beta band, pointing to changes in the EC distribution in AD.

In Figure 6, the regional EC averages in all the frequency bands are displayed. In most bands, the EC was highest in the parietal sensors, confirming the previously reported hub status of this region (Buckner et al., 2005; Tomasi and Volkow, 2011). In this band, temporal sensor EC values were relatively low in both groups, and were even lower in $\mathrm{AD}$ patients. This indicates a diminishing network role of those regions in the $\mathrm{AD}$ patients in the theta band. In the beta band, the parietal EC values were lower in AD, while the temporal and particularly occipital values were higher. In the gamma band, the hemispherical differences were marked, with lower frontal EC but higher EC values in the sensors over the left posterior hemisphere.

\section{$E C$ and cognition}

Finally, we assessed the correlations between regional EC values and MMSE score for the different frequency bands. The results are displayed in Table 3.

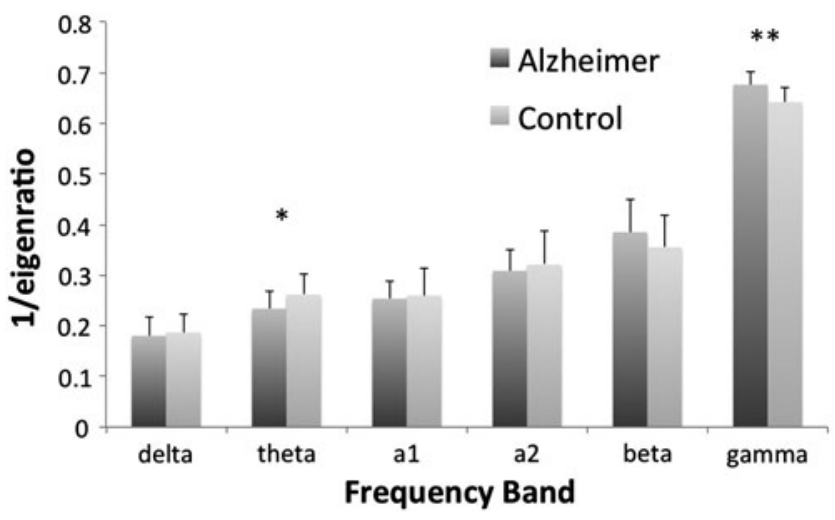

FIG. 5. Eigenratio results for the different frequency bands. It should be noted that the depicted results are based on the inverse of the original eigenratio (1/eigenratio) to obtain a value between 0 and 1 . Error bars indicate standard deviation. ${ }^{*} p<0.05,{ }^{* *} p<0.01$ (uncorrected). 


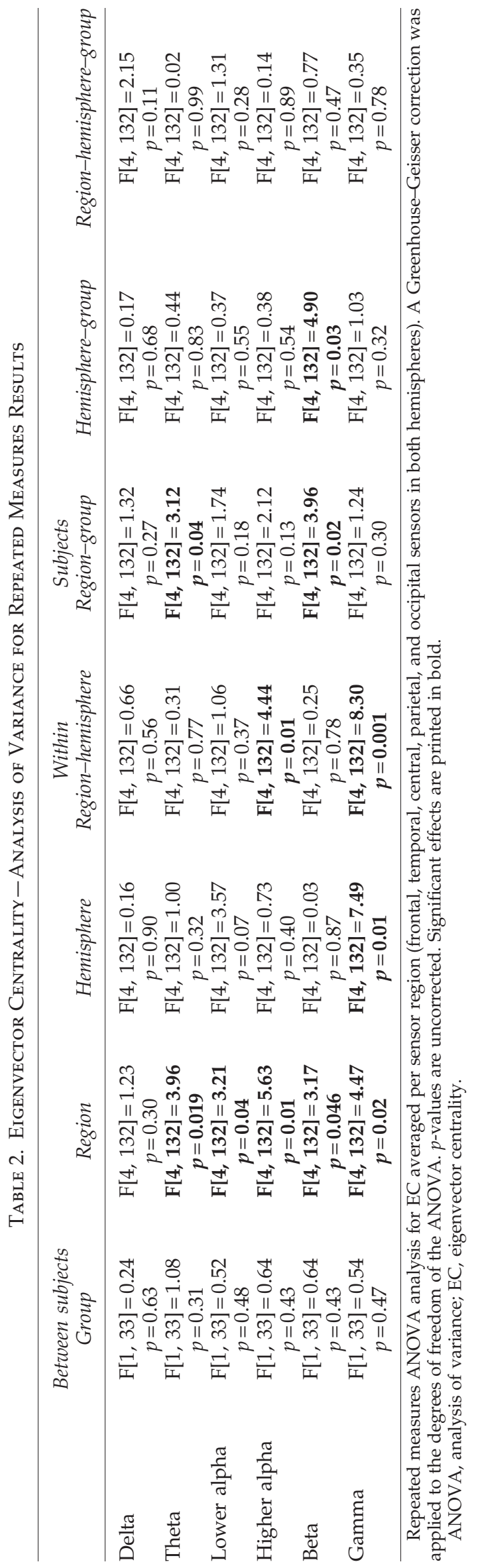

The left temporal regional EC in the theta band was strongly associated with the MMSE score in AD patients $(r=0.67, p=0.001)$ (see Fig. 7). In the other bands, the left temporal EC showed the same trend but with weaker, nonsignificant correlations. The right central EC in the theta band was negatively correlated to the MMSE score in AD patients $(r=-0.66, p=0.003)$, but not in the other bands.

In the lower alpha band, the pattern of EC values and changes in $\mathrm{AD}$ was similar. In the gamma band, the right parietal EC and MMSE were strongly correlated $(r=0.68$, $p=0.009)$.

\section{Discussion}

In this first application of GSA to MEG patient data, it is demonstrated that this technique is able to detect changes in the resting-state functional network integrity of earlystage Alzheimer patients. The main outcomes are a general loss of network integrity in the AD patients, especially in the higher frequency bands, and a distinct pattern of regional connectivity changes that are correlated to cognitive impairment. These findings are generally in line with previous literature and our hypotheses, although a few discrepancies were encountered as well.

\section{Global network topology}

The decreases in algebraic connectivity and spectral radius in several frequency bands in the AD patient group can be interpreted as a loss-of-network robustness and a deviation from the optimal configuration for dynamic processing. This agrees with related graph theoretical studies in AD that have been conducted so far (He et al., 2009; Stam, 2010), and supports the notion that in AD, a functional disconnection between regions is taking place, leading to suboptimal cognitive processing. The finding that these different methods point in the same direction provides a degree of validation. The results of topological and spectral graph measures cannot be directly compared, but the decrease in algebraic connectivity can be taken as a stronger and theoretically sounder sign of network breakdown than the previously reported loss of a small-world network structure. In the spectral approach, no previous model (e.g., Watts and Strogatz, 1998), normalization of graph measures (e.g., clustering coefficient and path length) through a comparison with random surrogate networks, or other additional methodological choices are required.

Theoretically, the relationship between network topology and network synchronizability is not straightforward (Arenas et al., 2008), and increased network stability might also be pathological, for example, by contributing to epileptic seizures. The prevalence of epilepsy in AD patients is higher than in the healthy population, and it is thought to be directly related to neurodegenerative pathophysiological processes (Larner, 2010; Palop and Mucke, 2009). The observed eigenratio decrease (increase of 1/eigenratio in Fig. 5) in the gamma band in AD indeed suggests higher network synchronization stability in AD. In the theta band, the eigenratio increases in AD patients, suggesting lower network synchronization stability in this band. This shift, contrary to the gamma-band findings, could also point to different network functions of the frequency bands, or to cross-frequency coupling effects; the theta-gamma-band relation with regard to memory processes 
Delta $(0.5-4 \mathrm{~Hz})$

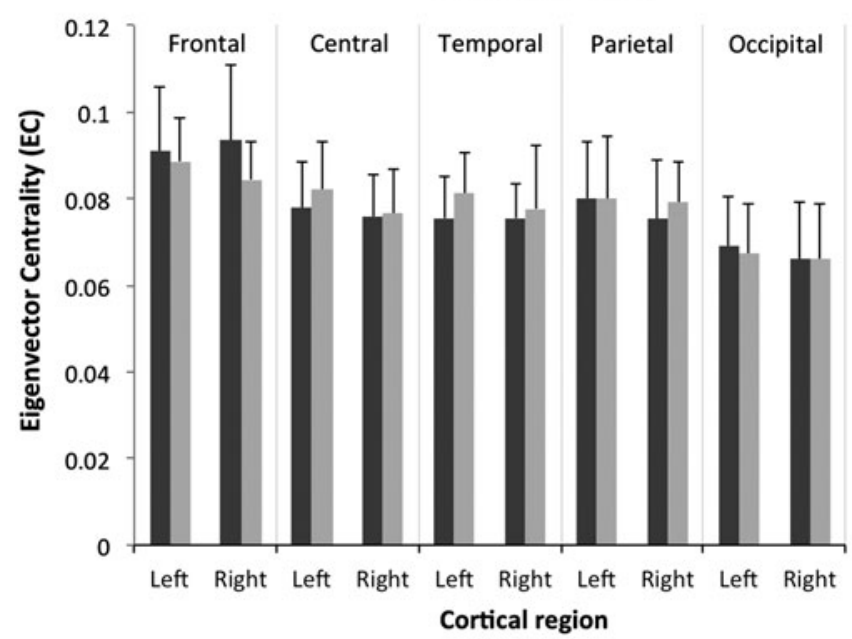

Lower alpha $(8-10 \mathrm{~Hz})$

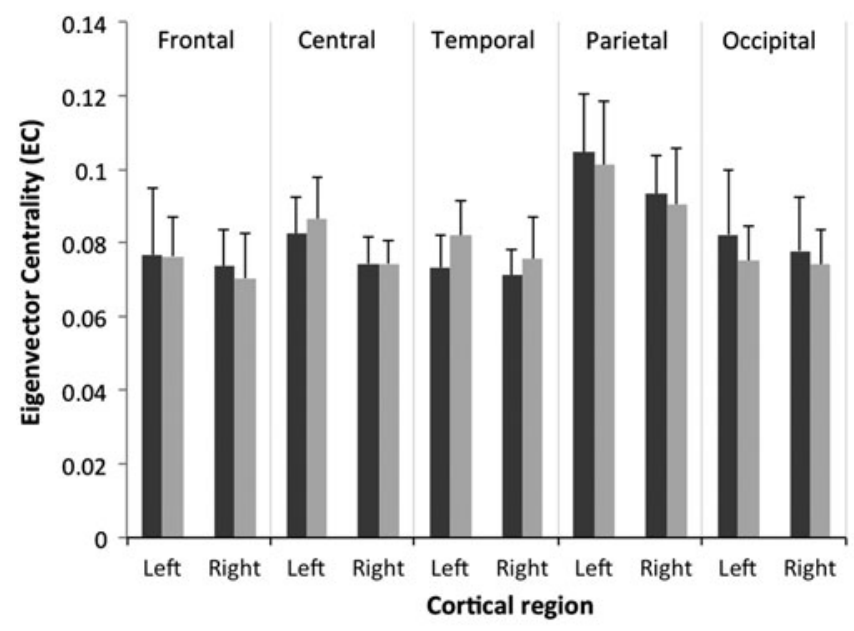

Beta $(13-30 \mathrm{~Hz})$

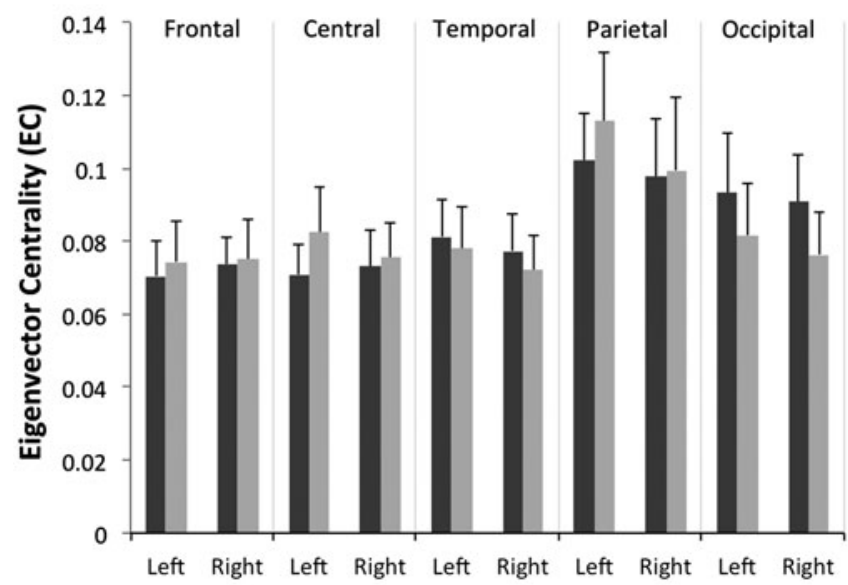

Cortical region

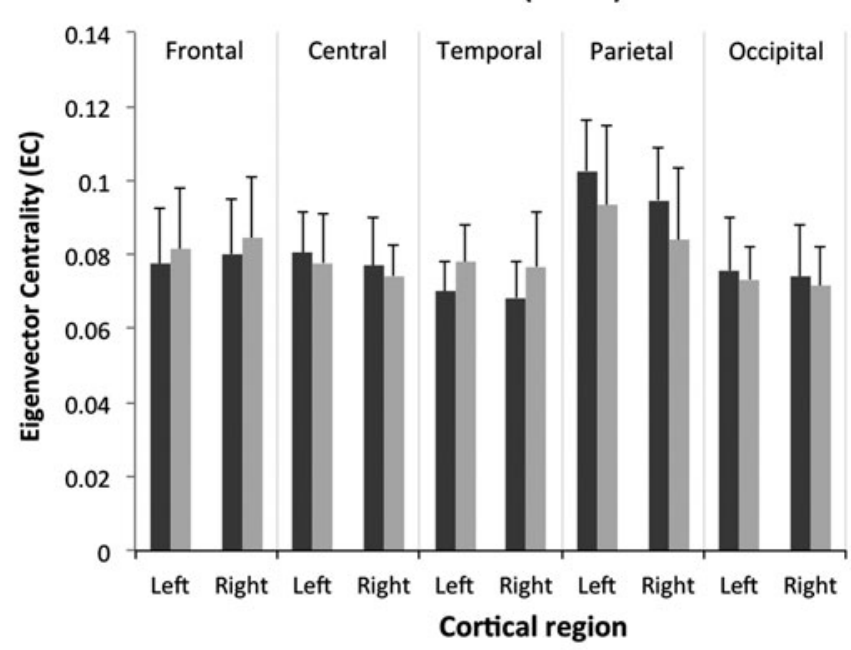

Higher alpha $(\mathbf{1 0 - 1 3} \mathrm{Hz})$

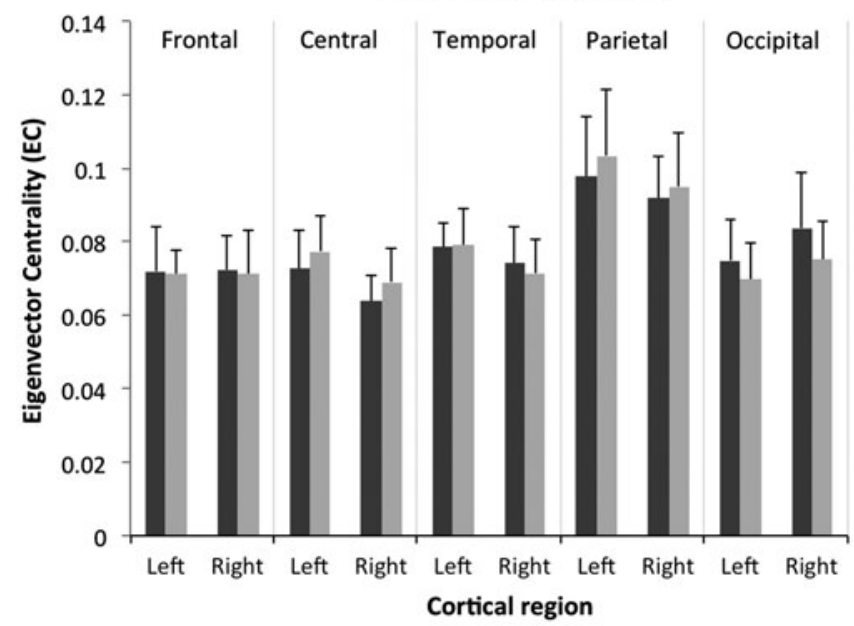

Gamma (30-45 Hz)

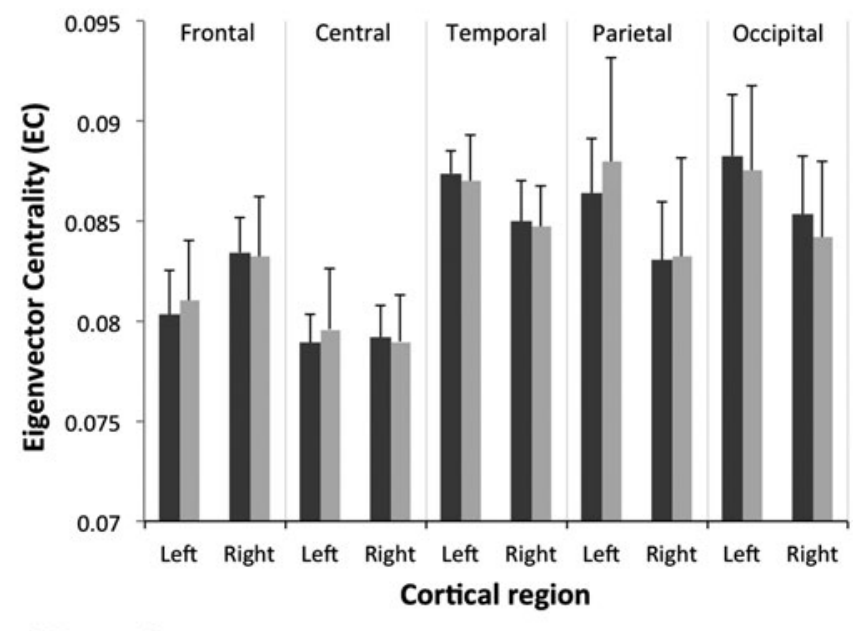

FIG. 6. Regional sensor-space ECM results for all frequency bands, Alzheimer patients versus control subjects. Error bars indicate standard deviation. ECM, eigenvector centrality mapping. 
Table 3. Relation Between Eigenvector Centrality and Mini Mental State Examination in Alzheimer's Disease Patients

\begin{tabular}{|c|c|c|c|c|c|c|}
\hline$E C$ & Delta & Theta & Lower alpha & Higher alpha & Beta & Gamma \\
\hline Left frontal & 0.03 & -0.02 & -0.25 & -0.25 & 0.09 & -0.18 \\
\hline Right frontal & -0.30 & 0.05 & -0.09 & -0.28 & -0.01 & -0.29 \\
\hline Left central & 0.09 & 0.27 & -0.14 & 0.15 & -0.05 & -0.14 \\
\hline Right central & -0.42 & $-0.66^{* *}$ & -0.26 & -0.16 & -0.04 & -0.16 \\
\hline Left temporal & 0.21 & $0.67^{* *}$ & $0.55^{*}$ & 0.38 & 0.25 & 0.26 \\
\hline Right temporal & 0.13 & -0.02 & 0.16 & 0.04 & 0.03 & -0.21 \\
\hline Left parietal & 0.14 & 0.32 & 0.47 & 0.21 & -0.33 & -0.07 \\
\hline Right parietal & -0.06 & -0.39 & 0.08 & 0.17 & -0.06 & $0.62 * *$ \\
\hline Left occipital & 0.22 & 0.19 & 0.00 & 0.02 & 0.12 & 0.30 \\
\hline Right occipital & 0.22 & -0.04 & -0.18 & 0.12 & 0.02 & 0.23 \\
\hline
\end{tabular}

Pearson's bivariate correlations between regional EC values in 10 sensor regions and MMSE score (AD patient group). Significant findings are printed in bold.

${ }^{*} p<0.05,{ }^{* *} p<0.01$ (uncorrected).

has been repeatedly described, and is very relevant in AD, where memory impairment is often the main symptom. On the other hand, the observed spectral gap decrease in the AD gamma band indicates that reaching a synchronized state will be harder for the network. This finding supports the loss of large-scale network synchronizability in AD.

\section{Regional EC}

In the present study, parietal sensors had the largest EC values in almost all frequency bands, characterizing them as main hub regions. This is in line with previous findings (Lo et al., 2010; Stam et al., 2009; Tomasi and Volkow, 2011), and with the presumed integrative function of the parietal association areas (Mesulam, 1998). In a recent functional MRI (fMRI) study, the EC was applied to restingstate voxel-based fMRI networks of healthy subjects to explore differences between individuals in various satiety states (Lohmann et al., 2010). Besides confirming the hub

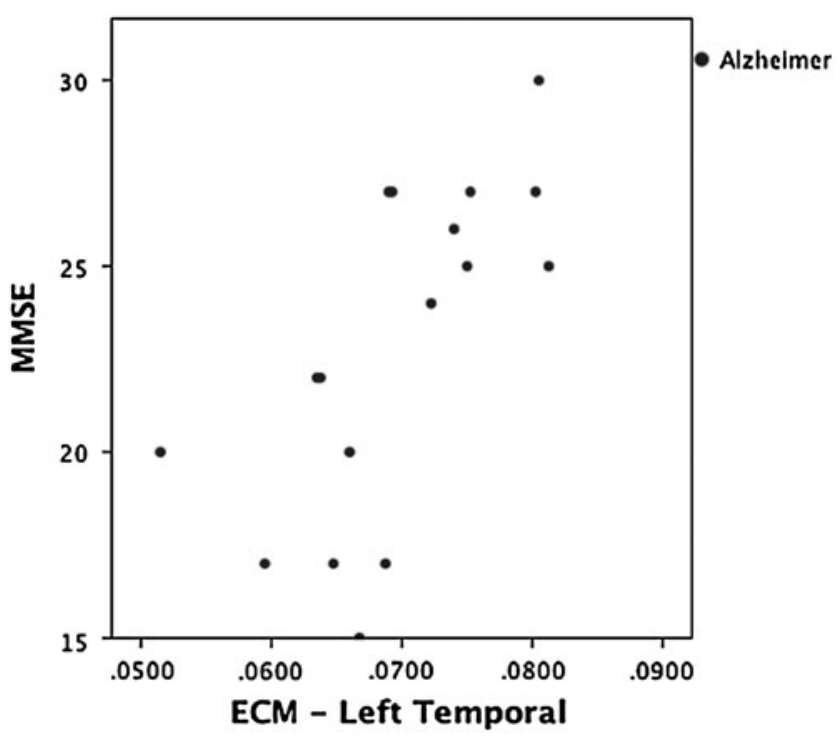

FIG. 7. Correlation between left temporal EC and MMSE score in the theta band (Alzheimer patient group only). $r=0.67, p=0.001$ (uncorrected). MMSE, mini mental state examination. status of the posterior cortical area, Lohmann and associates stress advantages such as the parameter- and assumptionfree nature of EC, as well as its computational efficiency compared with other centrality algorithms when investigating very large networks.

An intriguing recent insight that has emerged from network analysis in AD is that hub regions (especially parietal) are selectively vulnerable, and overlap strongly with regions of amyloid deposition, hypometabolism, and atrophy (Buckner et al., 2005; Drzezga et al., 2011). The vulnerability of parietal hub areas was reflected in our EC mapping findings by a parietal EC decrease in higher frequency bands and an increase in lower bands, that is, a loss of high frequency centrality. In addition, a notable regional EC change in $\mathrm{AD}$ took place in the theta band in both temporal areas, which already have a relatively low EC in healthy controls. On the one hand, this might be a sign of the known (medio) temporal atrophy and dysfunction in AD, and fit the observed decrease in theta band synchronizability. However, if a major part of the temporal connections are to and from the vulnerable parietal hub areas, then the EC decrease may be mainly due to the weakening of the parietal hub nodes. Thus, a possible explanation of this difference is the more indirect character of EC compared with degree centrality.

The striking correlation that was found in the theta band between the left temporal EC and MMSE score (see Table 3 and Fig. 7) suggests that the drop in the network centrality of the left temporal region in AD patients reflects the severity of cognitive symptoms. Overall, only a few clear correlations were found between the regional EC and MMSE score, even without a correction for multiple testing. Nevertheless, the temporal lobe association in the theta band is remarkably strong, and might hold most promise as a functional (bio-) marker of AD progression. It is well known that changes in theta activity are among the earliest neurophysiological signs that accompany AD, and in previous studies, left temporal lobe characteristics were specifically suggested as being $\mathrm{AD}$ disease progression markers (Fernandez et al., 2003; Gianotti et al., 2007; Osipova et al., 2005).

\section{Limitations and future directions}

Several potential limitations of this study should be taken into account. First of all, methodological choices might have 
influenced our outcome measures and subsequent interpretations: the use of resting-state data, the influence of volume conduction in MEG sensor-space analysis, and epoch selection. Another limitation could be our choice of the SL as a functional connectivity measure. Although we think that it is the most appropriate measure for our purpose given earlier SL-based studies, different functional connectivity measures could lead to different results. The influence of coupling measures on subsequent graph analysis results has not yet been investigated in a systematic way, but since a similar pattern of functional connectivity loss has been reported using different measures, we feel confident that the observed group differences cannot be explained by this choice. In this exploratory study, we opted for several commonly used and wellunderstood graph spectral measures that describe relevant properties with regard to brain network analysis. However, other measures, for example, describing network clustering properties, might be of special interest in future studies (Bialonski and Lehnertz, 2006). In addition, it would be interesting to compare the findings obtained in this study with the graph spectral results based on different functional connectivity measures, task-based datasets, or disease conditions.

From a clinical perspective, several limiting factors may have played a role: modest sample size, comorbidity, disease heterogeneity, and the use of psychoactive medication, as described in the methods section. However, since the occurrence of these phenomena were infrequent and distributed across both groups, it is not likely that they had a large influence on the observed group differences. People possessing any of these factors were not identified as outliers.

\section{Conclusion}

GSA detects changes in the resting-state functional network integrity of mild-to-moderate Alzheimer patients. The changes in AD patients point toward a less-efficient network configuration for dynamic processing. Moreover, the relationship between the loss of temporal lobe centrality and the cognitive impairment in $\mathrm{AD}$ indicates a potential value for tracking the disease course. These clinically relevant results, based on a solid, computationally efficient theoretical background that does not require a priori assumptions or arbitrary parameter settings, make GSA, in our opinion, a valid approach for exploring brain network integrity.

\section{Acknowledgments}

The authors thank Lieke Smits, Nicole Sistermans, Ellemarije Altena, Annelies van der Vlies, and Sofie Boom for neuropsychological assessments, and Karin Plugge and Ndedi Sijsma for performing the MEG recordings.

\section{Author Disclosure Statement}

No competing financial interests exist.

\section{References}

Arenas A, Díaz-Guilera A, Kurths J, Moreno Y, Zhou C. 2008. Synchronization in complex networks. Phys Rep 469:93-153. Bialonski S, Lehnertz K. 2006. Identifying phase synchronization clusters in spatially extended dynamical systems. Phys Rev E 74:051909.
Bonacich P. 2007. Some unique properties of eigenvector centrality. Social Netw 29:555-564.

Bonacich P, Lloyd P. 2001. Eigenvector-Like measures of centrality for asymmetric relations. Soc Netw 23:191-201.

Brouwer AE, Haemers WH. 2011. Spectra of graphs. Electronic Book. www.win.tue.nl/.aeb/ipm.pdf Accessed April 20, 2012.

Buckner RL, Snyder AZ, Shannon BJ, LaRossa G, Sachs R, Fotenos AF, et al. 2005. Molecular, structural, and functional characterization of alzheimer's disease: Evidence for a relationship between default activity, amyloid, and memory. J Neurosci 25:7709-7717.

de Haan W, Pijnenburg YA, Strijers RL, van der Made Y, van der Flier WM, Scheltens P, Stam CJ. 2009. Functional neural network analysis in frontotemporal dementia and alzheimer's disease using EEG and graph theory. BMC Neurosci 10:101.

de Haan W, van der Flier WM, Koene T, Smits LL, Scheltens P, Stam CJ. 2012. Disrupted modular brain dynamics reflect cognitive dysfunction in Alzheimer's disease. Neuroimage 59:3085-3093.

Deuker L, Bullmore ET, Smith M, Christensen S, Nathan PJ, Rockstroh B, Bassett DS. 2009. Reproducibility of graph metrics of human brain functional networks. Neuroimage 47:1460-1468.

Drzezga A, Becker JA, Van Dijk KR, Sreenivasan A, Talukdar T, Sullivan C, et al. 2011. Neuronal dysfunction and disconnection of cortical hubs in non-demented subjects with elevated amyloid burden. Brain 134 (pt. 6):1635-1646.

Dvorák Z, Mohar B. 2009. Spectral radius of finite and infinite planar graphs and of graphs of bounded genus. J Comb Theory Ser B 47:1096.

Farkas IJ, Derényi I, Barabási AL, Vicsek T. 2001. Spectra of "realworld" graphs: Beyond the semicircle law. Phys Rev E Stat Nonlin Soft Matter Phys 64(2 Pt 2):026704.

Fernandez A, Arrazola J, Maestu F, Amo C, Gil-Gregorio P, Wienbruch C, Ortiz T. 2003. Correlations of hippocampal atrophy and focal low-frequency magnetic activity in alzheimer disease: volumetric MR imaging-magnetoencephalographic study. Am J Neuroradiol 24:481.

Fiedler M. 1973. Algebraic connectivity of graphs. Czech Math J 23:298-305.

Folstein MF, Folstein SE, McHugh PR, Fanjiang G. 1983. Minimental state examination (MMSE). Arch Gen Psychiatry 40:812.

Gianotti LRR, Kunig G, Lehmann D, Faber PL, Pascual-Marqui RD, Kochi K, Schreiter-Gasser U. 2007. Correlation between disease severity and brain electric LORETA tomography in alzheimer's disease. Clin Neurophysiol 118:186-196.

He Y, Chen Z, Gong G, Evans A. 2009. Neuronal networks in alzheimer's disease. Neuroscientist 15:333-350.

Larner AJ. 2010. Epileptic seizures in AD patients. Neuromol Med 12:71-77.

Lo CY, Wang PN, Chou KH, Wang J, He Y, Lin CP. 2010. Diffusion tensor tractography reveals abnormal topological organization in structural cortical networks in alzheimer's disease. J Neurosci 30:16876-16885.

Lohmann G, Margulies DS, Horstmann A, Pleger B, Lepsien J, Goldhahn D, et al. 2010. Eigenvector centrality mapping for analyzing connectivity patterns in FMRI data of the human brain. Plos One 5:e10232.

McKhann G, Drachman D, Folstein M, Katzman R, Price D, Stadlan EM. 1984. Clinical diagnosis of alzheimer's disease: Report of the NINCDS-ADRDA work group under the auspices of department of health and human services task force on alzheimer's disease. Neurology 34:939-944. 
Mesulam MM. 1998. From sensation to cognition. Brain $121(\mathrm{Pt}$ 6):1013-1052.

Mohar B. 1991. The laplacian spectrum of graphs. Graph Theory Comb Appl 2:871-898.

Montez T, Linkenkaer-Hansen K, van Dijk BW, Stam CJ. 2006. Synchronization likelihood with explicit time-frequency priors. Neuroimage 33:1117-1125.

Newman MEJ. 2007. The mathematics of networks. In: Blume CE and Durlauf SN (eds.) The New Palgrave Encyclopedia of Economics. Basingstoke, UK: Palgrave Macmillan.

Osipova D, Ahveninen J, Jensen O, Ylikoski A, Pekkonen E. 2005. Altered generation of spontaneous oscillations in alzheimer's disease. Neuroimage 27:835-841.

Palop JJ, Mucke L. 2009. Epilepsy and cognitive impairments in Alzheimer disease. Arch Neurol 66:435-440.

Restrepo JG, Ott E, Hunt BR. 2005. Onset of synchronization in large networks of coupled oscillators. Phys Rev E 71:036151.

Rubinov M, Knock SA, Stam CJ, Micheloyannis S, Harris AW, Williams LM, Breakspear M. 2009. Small-World properties of nonlinear brain activity in schizophrenia. Hum Brain Mapp 30:403-416.

Rubinov M, Sporns O. 2010. Complex network measures of brain connectivity: Uses and interpretations. Neuroimage 52:10591069.

Rubinov M, Sporns O. 2011. Weight-Conserving characterization of complex functional brain networks. Neuroimage 56:2068-2079.

Rulkov NF, Sushchik MM, Tsimring LS, Abarbanel HD. 1995. Generalized synchronization of chaos in directionally coupled chaotic systems. Phys Rev E Stat Phys Plasmas Fluids Relat Interdiscip Topics 51:980-994.

Sanz-Arigita EJ, Schoonheim MM, Damoiseaux JS, Rombouts SA, Maris E, Barkhof F, et al. 2010. Loss of 'small-world' networks in alzheimer's disease: Graph analysis of FMRI resting-state functional connectivity. Plos One 5:e13788.

Sporns O. 2010. Networks of the brain. Cambridge, MA: The MIT Press.

Stam CJ. 2010. Characterization of anatomical and functional connectivity in the brain: A complex networks perspective. Int J Psychophysiol 77:186-194.

Stam CJ, de Haan W, Daffertshofer A, Jones BF, Manshanden I, van Cappellen van Walsum AM, et al. 2009. Graph theoretical analysis of magnetoencephalographic functional connectivity in alzheimer's disease. Brain 132(Pt 1):213-224.
Stam CJ, Dijk BWV. 2002. Synchronization likelihood: An unbiased measure of generalized synchronization in multivariate data sets. Physica D Nonlin Phenomena 163:236-251.

Stam CJ, Jones BF, Nolte G, Breakspear M, Scheltens P. 2007. Small-World networks and functional connectivity in alzheimer's disease. Cereb Cortex 17:92-99.

Supekar K, Menon V, Rubin D, Musen M, Greicius MD. 2008. Network analysis of intrinsic functional brain connectivity in alzheimer's disease. Plos Comput Biol 4:e1000100.

Tomasi D, Volkow ND. 2011. Functional connectivity hubs in the human brain. Neuroimage 57:908-917.

Takens F. 1981. Detecting strange attractors in turbulence. Lect Notes Math 898:366-381.

Theiler J. 1986. Spurious dimension from correlation algorithms applied to limited time-series data. Phys Rev A 34:24272432.

Van Mieghem P. 2011. Graph spectra for complex networks. Cambridge, MA: Cambridge University Press.

Van Mieghem P, Omic JS, Kooij RE. 2009. Virus Spread in Networks. IEEE/ACM Transaction on Networking, Vol. 17, No. 1, February, pp 1-14.

van Wijk BC, Stam CJ, Daffertshofer A. 2010. Comparing brain networks of different size and connectivity density using graph theory. Plos One 5:e13701.

Verhage F. 1965. Intelligence and age in a dutch sample. Human Development 8:238-245.

Vrba J, Robinson SE. 2001. Signal processing in magnetoencephalography. Methods 25:249-271.

Watts DJ, Strogatz SH. 1998. Collective dynamics of 'smallworld' networks. Nature 393:440-442.

Address correspondence to: Willem de Haan

Department of Neurology Alzheimer Center

VU University Medical Center P.O. Box 7057

1007 MB Amsterdam The Netherlands

E-mail: w.dehaan@vumc.nl

\section{Appendix}

\section{Mathematical Background of Synchronization Likelihood}

The synchronization likelihood (SL) is a measure of the generalized synchronization between two dynamical systems $X$ and $Y$ (Stam and Dijk, 2002). A generalized synchronization (Rulkov et al., 1995) exists between $X$ and $Y$ of the state-ofthe-response system and is a function of the driver system: $Y=F(X)$. The first step in the computation of the SL is to convert the time series $x_{\mathrm{i}}$ and $y_{\mathrm{i}}$ recorded from $X$ and $Y$ as a series of state-space vectors using the method of time-delay embedding (Takens, 1981):

$$
X_{i}=\left(x_{i}, x_{i+L}, x_{i+2 \times L}, x_{i+3 \times L \ldots,}, x_{i+(m-1) \times L}\right)
$$

where $L$ is the time lag, and $m$ is the embedding dimension.
From a time series of $N$ samples, $N-(m \times L)$ vectors can be reconstructed. State-space vectors $Y_{\mathrm{i}}$ are reconstructed in the same way.

Synchronization likelihood is defined as the conditional likelihood that the distance between $Y_{i}$ and $Y_{j}$ will be smaller than a cutoff distance $r_{y}$, given that the distance between $X_{i}$ and $X_{j}$ is smaller than a cutoff distance $r_{x}$. In the case of maximal synchronization, this likelihood is 1 ; in the case of independent systems, it is a small, but nonzero, number, namely $P_{\text {ref. }}$ This small number is the likelihood that two randomly chosen vectors $Y$ ( or $X$ ) will be closer than the cutoff distance $r$. In practice, the cutoff distance is chosen such 
that the likelihood of the random vectors being close is fixed

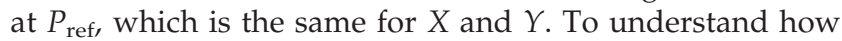
$P_{\text {ref }}$ is used to fix $r_{x}$ and $r_{y}$, we first consider the correlation integral:

$$
C_{r}=\frac{2}{N(N-w)} \sum_{i=1}^{N} \sum_{j=i+w}^{N-w} \theta\left(r-\left|X_{i}-X_{j}\right|\right)
$$

Here, the correlation integral $C_{r}$ is the likelihood that two randomly chosen vectors $X$ will be closer than $r$. The vertical bars represent the Euclidean distance between the vectors. $N$ is the number of vectors, $\mathrm{w}$ is the Theiler correction for autocorrelation (Theiler, 1986), and $\theta$ is the Heaviside function: $\theta(X)=0$ if $X \geq 0$, and $\theta(X)=1$ if $X<0$. Now, $r_{x}$ is chosen such that $\mathrm{Cr}_{\mathrm{X}}=$ $P_{\text {ref }}$ and $r_{y}$ is chosen such that $\mathrm{Cr}_{y}=P_{\text {ref. }}$. The SL between $X$ and $Y$ can now be formally defined as:

$$
\begin{aligned}
S L= & \frac{2}{N(N-w) P_{\text {ref }}} \\
& \times \sum_{i=1}^{N} \sum_{j=i+w}^{N-w} \theta\left(r_{x}-\left|X_{i}-X_{j}\right|\right) \theta\left(r_{y}-\left|Y_{i}-Y_{j}\right|\right)
\end{aligned}
$$

$\mathrm{SL}$ is a symmetric measure of the strength of synchronization between $X$ and $Y\left(S L_{X Y}=S L_{Y X}\right)$. In equation (3), the averaging is done all over $i$ and $j$; by doing the averaging only over $j$, SL can be computed as a function of time $i$. From equation (3), it can be seen that in the case of complete synchronization, $\mathrm{SL}=1$; in the case of complete independence, $\mathrm{SL}=P_{\text {ref. }}$. In the case of intermediate levels of synchronization, $P_{\text {ref }}<\mathrm{SL}<1$. In the present study, the following parameters were used: $P_{\text {ref }}$ was set at 0.01 , for the state space embedding a time lag of 10 samples, an embedding dimension of 10, and a Theiler correction (W2) of 0.1 . 